Schmerz 2010 $24: 605-612$

DOI 10.1007/s00482-010-0989-9

Online publiziert: 30. Oktober 2010

(c) Deutsche Gesellschaft zum Studium des Schmerzes. Published by Springer-Verlag all rights reserved 2010

L. Bertram ${ }^{1,2} \cdot$ S. Stiel ${ }^{1}$. F. Elsner ${ }^{1} \cdot$ L. Radbruch ${ }^{3} \cdot$ A. Davies ${ }^{4} \cdot$ F. Nauck ${ }^{5}$.

B. Alt-Epping ${ }^{5}$

${ }^{1}$ Klinik für Palliativmedizin, RWTH Aachen

${ }^{2}$ Klinik für Anästhesiologie, RWTH Aachen

${ }^{3}$ Klinik für Palliativmedizin, Universitätsklinikum Bonn; Zentrum für Palliativmedizin, Malteser Krankenhaus, Bonn

${ }^{4}$ Palliative Care Department, The Royal Marsden NHS Foundation Trust, London, UK

${ }^{5}$ Abteilung Palliativmedizin, Universitätsmedizin Göttingen

\title{
Erfahrungen von Tumorpatienten mit Durchbruchschmerzen und medikamentösen Behandlungen
}

In der Klassifikation wird maßgeblich zwischen 2 Auslösemomenten des Durchbruchschmerzes unterschieden: Ereignisabhängige, vorhersehbare Auslöser wie Bewegung bzw. ereignisabhängige, unvorhersehbare Auslöser, wie z. B. Husten oder Niesen, werden bei 40-60\% der Durchbruchschmerzepisoden beobachtet. Spontane Auslöser für Durchbruchschmerzen sind bei $20-60 \%$ der Episoden ursächlich. Durchbruchschmerzen, die durch ein „end-of-dose-failure“ der Basismedikation zur Dauerschmerztherapie ausgelöst werden, werden dabei nicht berücksichtigt $[4,15,21,27]$.

In der palliativmedizinischen Literatur werden Prävalenzen des Durchbruchschmerzes zwischen 23 [5] und 93\% [23] aller Tumorpatienten berichtet, wobei sich im Durchschnitt der bisher publizierten Ergebnisse Anteile von ca. 6070\% schätzen lassen.

Durchbruchschmerzen haben für Patienten eine verminderte Zufriedenheit mit ihrer Schmerztherapie $[19,29]$, eine verminderte Lebensqualität [1] und eine vermehrte Angst vor körperlichem Leid und vor einem qualvollen Sterben [19] zur Folge. Zusätzlich entstehen höhere Gesundheitskosten durch vermehrte und längere Krankenhausaufenthalte der betroffenen Patienten $[1,5]$.

In den letzten Jahren wurden zahlreiche Studien durchgeführt, um einerseits die klinischen Charakteristika des Durchbruchschmerzes weiter zu definie- ren und andererseits Empfehlungen zur klinischen Therapie zu entwickeln. Diese Forschungsbemühungen konzentrierten sich auf das Ziel, Aussagen über die Eigenschaften eines optimalen zukünftigen Medikaments zur Durchbruchschmerztherapie zu treffen. Hier spielten nicht nur rein pharmakologische Wirkeigenschaften und Applikationswege bedeutende Rollen, sondern auch subjektive Patientenpräferenzen und -erfahrungen sowie ökonomische Argumente im Sinne allgemeiner Gesundheitskosten, z. B. durch (verlängerte) Krankenhausaufenthalte der betroffenen Patienten und durch die Preisgestaltung dieser Analgetika.

In der Behandlung von Durchbruchschmerz müssen dessen besondere Merkmale berücksichtigt werden. Betroffene Patienten berichten sehr hohe Schmerzintensitäten auf numerischen Rangskalen (NRS) mit einer medianen Zeit von 3 min bis zum Erreichen der absoluten Schmerzspitze $[10,16,17]$. Bei einer mittleren Schmerzdauer von 30 min [6] und mittleren Häufigkeiten von 4 Episoden am Tag [18] wird bei 31\% der Episoden ein Rückgang nach weniger als $15 \mathrm{~min}$ bzw. bei $64 \%$ der Episoden nach $30 \mathrm{~min}$ $[6,15]$ auch ohne medikamentöse Interventionen erlebt.

Die beschriebenen Charakteristika von Durchbruchschmerzen verlangen folglich

L. Bertram und S. Stiel haben in gleichem Umfang zu diesem Manuskript beigetragen. genannten vorübergehenden Schmerzexazerbationen hinzukommen [4]. 
nach einer möglichst schnell wirksamen, kurz anhaltenden, nicht- oder nur minimal-invasiven, praktikablen, nebenwirkungsarmen und preiswerten Therapie, die selbstständig vom Patienten angewendet werden kann. In den bisher verfügbaren Behandlungsempfehlungen wird an erster Stelle, sofern möglich, das Ansetzen einer kausalen Therapie und im zweiten Schritt eine Bedarfsmedikation von ein Zehntel bis ein Sechstel der Tagesdosis der Dauermedikation für die Behandlung des Durchbruchschmerzes angeraten oder eine individuelle Dosisfindung per Titration diskutiert [8]. Eine Erhöhung der Daueranalgesie könnte zwar präventiv dem Durchbruchschmerz entgegenwirken, bietet aber durch die Gefahr der chronischen Überdosierung mit Nebenwirkungen wie Sedierung, Übelkeit, Verstopfung oder sogar Atemdepression keine Alternative zur Durchbruchanalgesie. Vorsichtige Dosisanpassungen werden jedoch bei einzelnen Patienten mit Durchbruchschmerzepisoden als effektiv beschrieben [11].

Aus der klinischen Erfahrung zeigt sich jedoch, dass verfügbare Analgetika oft einen verzögerten Wirkeintritt und eine zu lange Wirkdauer aufweisen, als dass z. B. eine in wenigen Minuten auftretende und weniger als $1 \mathrm{~h}$ anhaltende Schmerzspitze aus Patientensicht zufriedenstellend gelindert werden könnte [26]. Dementsprechend konzentrieren sich die aktuellen Forschungsbemühungen auf Präparate und Applikationsformen für Medikamente bei Durchbruchschmerzen, die diesen Anforderungen entsprechen.

Die vorliegende Untersuchung soll einen Beitrag dazu leisten, das Erleben von Durchbruchschmerzen, bisher erprobte Therapiestrategien sowie subjektive Präferenzen und Erwartungen von Patienten mit Durchbruchschmerzen an ein optimales, zukünftiges Schmerzmedikament zu explorieren.

\section{Methode}

Die hier beschriebene Untersuchung ist Teil eines multizentrischen europäischen Kooperationsprojektes „European Survey of Oncology Patients' Experience of Breakthrough Pain“ mit 9 Zentren aus Großbritannien, Schweden, Dänemark und Deutschland unter Federführung des Royal Marsden Hospitals in Sutton, Großbritannien [2].

Für die vorliegende Auswertung wurden die Patienten der beiden deutschen Studienzentren ausgewertet. In jedem der teilnehmenden Zentren sollten in einer 6-monatigen Erhebungsphase Tumorpatienten, die von der Abteilung Palliativmedizin primär verantwortlich oder konsiliarisch betreut wurden, mithilfe eines Fragebogens zu ihren Erfahrungen mit Durchbruchschmerzen und über ihre Einstellungen zu verfügbaren und aktuell entwickelten Therapien für Durchbruchschmerzen befragt werden.

In einem ersten Teil des Fragebogens wurden 5 Screeningfragen zu Ein- und Ausschlusskriterien und 6 Fragen zu demografischen und krankheitsbezogenen Daten gestellt, um Patienten mit Durchbruchschmerz von Patienten mit unzureichend kontrolliertem Dauerschmerz zu unterscheiden.

Im zweiten Fragebogenteil wurden insgesamt 39 Items aus den folgenden $5 \mathrm{Ab}$ schnitten erfragt:

- A) Charakteristika des Durchbruchschmerz,

- B) Daueranalgesie,

- C) Durchbruchanalgesie,

- D) Wichtigste erwünschte Merkmale für neue Therapien gegen Durchbruchschmerzen,

- E) Bisherige Erfahrungen mit alternativen Aufnahmewegen der Medikamenteneinnahme bei Durchbruchschmerzen bzw. anderen Problemen mit den jeweiligen Kontraindikationen und Problemen bei der Anwendung sowie das hypothetische In-Betracht-Ziehen dieser Applikationswege.

Die Fragebögen wurden anonymisiert und zentral durch die koordinierende Einrichtung ausgewertet, aufbewahrt und in eine elektronische Datenbank eingegeben.

\section{Studiendesign}

In der Zeit von 24.11.2008 bis 12.02.2010 wurden stationäre, ambulante und konsiliarisch behandelte Patienten mit Tumorerkrankungen der Klinik für Palliativmedizin des Universitätsklinikums Aa- chen und der Abteilung für Palliativmedizin des Universitätsklinikums Göttingen um deren Teilnahme an dieser Studie gebeten. Nach ausführlicher Patienteninformation erteilten Patienten ihre schriftliche Einwilligung zur Teilnahme. Dieser Studie wurde von der Ethikkommission der Medizinischen Fakultät der RWTH Aachen und der Ethikkommission der Universitätsmedizin Göttingen zugestimmt.

In diese Fragebogenstudie wurden $\mathrm{Pa}$ tienten älter als 18 Jahre mit einer Tumordiagnose aufgenommen, die im Zusammenhang mit ihrer Tumorerkrankung an Schmerzen leiden und deren Schmerzen die meiste Zeit vorhanden (während der letzten Woche $\geq 12 \mathrm{~h} / \mathrm{Tag}$ ) sind oder vorhanden wären, wenn sie kein Schmerzmanagement erhielten. Zudem sollten diese Schmerzen die meiste Zeit unter Kontrolle sein und für $\geq 12 \mathrm{~h} /$ Tag in der letzten Woche als „keine“ oder „leicht", aber nicht als „mäßig“" oder „schwer“ beurteilt werden. Als weitere Einschlusskriterien wurden das Empfinden einer kurzzeitigen Schmerzverstärkung (= tumorbedingter Durchbruchschmerz) und der regelmäßige Gebrauch von starken Schmerzmitteln (hochpotente Opioide wie Morphium, Fentanyl, Hydromorphon, Methadon, Oxycodon) gegen die andauernden Schmerzen (= Dauerschmerz) definiert. Eine primäre Durchbruchanalgesie war für die Befragten zum Zeitpunkt der Studienerhebung nicht zwingend notwendig.

\section{Ergebnisse}

In der Zeit von Februar 2009 bis Januar 2010 nahmen jeweils 40 stationäre Tumorpatienten der Klinik für Palliativmedizin der RWTH Aachen und der Abteilung für Palliativmedizin des Universitätsklinikums Göttingen an der Erhebung teil. Die Geschlechterverteilung war etwa ausgeglichen (42 Männer, 38 Frauen); die 8o Studienteilnehmer waren im Mittel 60,5 Jahre alt (33-91 Jahre) und litten unter Tumoren des Verdauungstraktes (29\%), der Lunge (16\%), der Brust (14\%), mit unbekanntem Primärtumor (13\%) und des urologischen Traktes (10\%). Der Funktionsstatus war bei $42 \%$ der Patienten stark eingeschränkt (ECOG 3 oder 4 ). 
Schmerz 2010 - 24:605-612 DOI 10.1007/s00482-010-0989-9

(c) Deutsche Gesellschaft zum Studium des Schmerzes. Published by Springer-Verlag - all rights reserved 2010

\section{Bertram · S. Stiel · F. Elsner · L. Radbruch · A. Davies · F. Nauck · B. Alt-Epping Erfahrungen von Tumorpatienten mit Durchbruchschmerzen und medikamentösen Behandlungen}

\section{Zusammenfassung}

Hintergrund. In der Palliativmedizin berichten ca. $80 \%$ aller Krebspatienten über tumorbedingte Schmerzen, und bis zu $80 \%$ dieser Patienten haben Durchbruchschmerzen. Die vorliegende Untersuchung exploriert das Erleben von Durchbruchschmerz, die Erfahrungen mit bisherigen Therapiestrategien und die Erwartungen an ein optimales Schmerzmedikament aus der Sicht von Tumorpatienten in Deutschland.

Methode. Im Rahmen des „European Survey of Oncology Patients' Experience of Breakthrough Pain" wurden von November 2008 bis Februar 201080 stationäre und ambulante Tumorpatienten zweier Palliativkliniken in Deutschland mit einem standardisierten Fragebogen zu den Charakteristika ihres Durchbruchschmerzes, zur Schmerzmedikation, zu ihren Erwartungen an die Therapie und zu ihren Erfahrungen mit verschiedenen Applikationswegen von Analgetika befragt. Ergebnisse. Die Studienteilnehmer beschrieben 1-12 Episoden ereignisabhängiger (47,5\%) bzw. spontaner (37,5\%) Durchbruchschmerzepisoden pro Tag, die in $71 \%$ der Fälle als "schwer" eingestuft wurden und die allgemeine Aktivität, Stimmung, das Gehvermögen und die normale Arbeit stark einschränkten. Insgesamt 64\% der Teilnehmer fanden Erleichterung des Durchbruchschmerzes durch medikamentöse (26\%) und nicht-medikamentöse (73\%) Maßnahmen. Subkutan und oral applizierbare Präparate fanden häufig Zustimmung (40 bzw. 39\%), während Möglichkeiten intranasaler oder inhalativer Medikamentenverabreichungen zurückhaltend angewendet wurden (je 1,25\%). Lediglich 64\% der Teilnehmer zeigten sich zu- frieden mit der Therapie inrer Durchbruchschmerzen.

Schlussfolgerungen. Die Therapie von Durchbruchschmerz scheint weiterhin suboptimal zu sein. Möglicherweise sind Behandlungsstandards zur Verbesserung der Schmerzlinderung für betroffene Patienten nicht bekannt und etabliert genug, sodass sie derzeit keine konsequente Umsetzung finden. Unter Beachtung der Schmerzursachen und einer multiprofessionellen Behandlung gilt es in der Therapie von Durchbruchschmerz, die in verschiedenen Applikationsformen verfügbaren, schnell wirksamen Präparate sinnvoll anzuwenden.

Schlüsselwörter

Palliativmedizin · Tumorpatient .

Durchbruchschmerz · Applikation · Opioide

\section{Experiences of cancer patients with breakthrough pain and pharmacological treatments}

\section{Abstract}

Background. Of cancer patients receiving palliative care, $80 \%$ suffer from cancer pain, and again $80 \%$ of these patients report breakthrough pain. This study explores the patients' perception of breakthrough pain, their experiences with existing therapeutic regimens and their expectations regarding an ideal breakthrough pain medication. Method. From November 2008 to February 2010 two German palliative care units recruited 80 in- or outpatient cancer patients who completed a standardized questionnaire on breakthrough pain characteristics, analgesic medication, attitudes towards new treatment approaches for breakthrough pain, and experiences with alternative routes of drug ad- ministration as part of the "European Survey of Oncology Patients' Experience of Breakthrough Pain".

Results. The study participants suffered from 1-12 episodes of either incident (47.5\%) or spontaneous pain (37.5\%) per day which were perceived as "severe" in $71 \%$ of all cases. These exacerbations highly interfered with the patients' general activity, mood, walking ability, and normal work. Overall, $64 \%$ of the patients reported alleviation from pharmacological (26\%) and non-pharmacological $(73 \%)$ interventions. Subcutaneous (40\%) and oral (39\%) routes were used frequently; intranasal (1.25\%) and intrapulmonary $(1.25 \%)$ routes were used rarely. Only $64 \%$ of all participants stated an overall satisfaction with their breakthrough analgesia.

Conclusion. The diagnosis and treatment of breakthrough pain seems to be conducted in a suboptimal manner, and standard recommendations on breakthrough pain relief are not implemented consistently. Possible causes of pain should be taken into account as well as multi-professional treatment interventions and alternative routes of administration of fast onset, effective drugs should be considered.

\section{Keywords}

Palliative care - Cancer patients .

Breakthrough pain · Application - Opioids 


\section{Charakteristika der \\ Durchbruchschmerzen}

\section{Häufigkeit, Auslöser und Dauer der Schmerzepisoden}

Die teilnehmenden Patienten berichten zwischen 1 und 12 Schmerzepisoden (im Mittel 4) pro Tag, die entweder ereignisabhängig (47,5\%), spontan durch unvorhersehbare Faktoren bedingt (37,5\%) oder in Kombination beider Gründe (15\%) auftraten. Die Schmerzintensitäten dieser Episoden wurden von $71 \%$ der Studienteilnehmer als „schwer" eingestuft, von $25 \%$ als „mittel“ und von $3 \%$ als , "leicht" bei $1 \%$ fehlenden Angaben. Bei ereignisabhängigen Episoden wurde eine mediane Zeit bis zur Schmerzspitze von $5 \mathrm{~min}$ angegeben (o-240 min, Mittel $22 \mathrm{~min}$ ), während nach spontaner Auslösung der Aufbau einer Schmerzepisode im Median 10 min dauerte (o-18o min, Mittel $24 \mathrm{~min}$ ).

\section{Linderung und Folgen des Durchbruchschmerzes}

Ein Teil der Patienten $(\mathrm{n}=43)$ berichtete, dass eine Durchbruchschmerzepisode im Median 20 min (o-48o min, im Mittel $64 \mathrm{~min}$ ) andauert, bis sie ohne Behandlung nachlässt. Bei $64 \%$ der Befragten werden wirksame Maßnahmen zur Schmerzreduktion eingesetzt, bei $21 \%$ der Befragten beenden die jeweils eingesetzten Maßnahmen die Durchbruchschmerzepisode nicht bzw. in 14\% nur manchmal (1\% fehlende Angaben). Diese Maßnahmen umfassten jeweils medikamentöse (26\%) oder nicht-medikamentöse (73\%) Therapien oder eine Kombination aus beiden (1\%).

Die Mehrheit der Teilnehmer (84\%) fühlte sich durch den Durchbruchschmerz in ihren Aktivitäten beeinträchtigt. Auf numerischen Rangskalen zwischen o (= keine) bis 10 (= vollständige Beeinträchtigung) gaben die Patienten im Mittel Auswirkungen von 7 in Bezug auf ihre allgemeine Aktivität, ihre Stimmung und das Gehvermögen an, 8 auf normale Arbeit, jeweils 6 auf ihren Schlaf, 6,5 auf die Lebensfreude sowie 5 auf Beziehungen $\mathrm{zu}$ anderen Menschen.

\section{Daueranalgesie und}

Durchbruchanalgesie

Zur regelmäßigen Therapie des Dauerschmerzgeschehens sowie auch zur Behandlung von Durchbruchschmerz nutzten die befragten Patienten am häufigsten Fentanyl oder Hydromorphon (• Tab. 1). Die Dosierung der Durchbruchschmerzmedikation ließ sich im Mittel als ein Viertel der Tagesdosis der Dauermedikation berechnen mit einer Spannweite zwischen 6 und $33 \%$.

\section{Linderung durch Durchbruchanalgesie}

Die erzielte Schmerzlinderung durch die Durchbruchanalgesie wurde von $14 \%$ als vollständig, $59 \%$ als gut, $15 \%$ als leicht und von $3 \%$ ohne Schmerzlinderung (bei 10\% fehlenden Angaben) eingestuft. Die Patienten schätzten die Dauer des Wirkbeginns der individuellen Durchbruchanalgesie auf $20 \mathrm{~min}$ im Mittel (1-180 min); bis zur vollständigen Entfaltung der Wirkung vergingen im Mittel 40 min (2-240 min).

\section{Nebenwirkungen und Anwendungsprobleme der Durchbruchanalgesie}

Die Hälfte aller Teilnehmer (50\%) gab keine unerwünschten Nebenwirkungen durch die Durchbruchanalgesie an. Insgesamt $45 \%$ aller Teilnehmer beklagten mindestens 1 Nebenwirkung, v. a. Schläfrigkeit $(n=19)$ und Verstopfung $(n=14)$, seltener Übelkeit und Erbrechen $(n=5)$, Mundtrockenheit $(n=4)$, Schwindel, Sehstörungen und Anorexie jeweils bei $2 \mathrm{~Pa}$ tienten und andere Symptome, wie z. B. Diarrhö, Stimmungsschwankungen und Konzentrationsstörungen, jeweils 1 Patient.

Nur 3 Patienten berichteten über andere, anwendungsbezogene Probleme der Durchbruchanalgesie wie schlechter Geschmack (2 Patienten) und praktische Anwendungsprobleme (1 Patient).

\section{Zufriedenheit mit und Barrieren der Durchbruchanalgesie}

Die Mehrheit der Patienten (64\%) zeigte sich zufrieden mit ihrer Durchbruchanalgesie, 34\% waren unzufrieden (fehlende Angabe 3 Patienten); 56\% al-
Hier steht eine Anzeige.

Springer 
ler Teilnehmer gaben an, jede Episode des Durchbruchschmerzes mit starken Schmerzmitteln zu behandeln, $43 \%$ taten dies nicht (fehlende Angabe 1 Patient). $\mathrm{Zu}$ den häufigsten Gründen der betroffenen Patienten, Durchbruchanalgetika nicht anzuwenden, zählten die Angst, von dem Medikament abhängig zu werden (8 Patienten), sich an das Schmerzmittel zu gewöhnen (7 Patienten) oder vor Nebenwirkungen der Schmerzmittel (4 Patienten). Noch häufiger wurde als Grund gegen die Bedarfsmedikation angeführt, dass die Durchbruchschmerzepisoden nicht immer lange anhielten (17 Patienten) oder nicht immer schwer waren (12 Patienten), andere Maßnahmen effektiv waren oder die Schmerzmittel keine Wirkung zeigten (3 Patienten). Ein Patient gab an, dass der Arzt oder die Krankenschwester der Anwendung von Schmerzmitteln Grenzen gesetzt hatte.

\section{Neue Therapien für \\ Durchbruchschmerz}

In der Abwägung der Wichtigkeit von 6 Merkmalen entschieden sich die Patienten in einer Rangliste mehrheitlich dafür, dass eine neue Therapie zur Behandlung von Durchbruchschmerzen an erster Stelle eine vollständige $(n=48)$ und an zweiter Stelle eine schnelle Schmerzlinderung $(n=43)$ erzielen sollte ( $\bullet$ Tab. 2$)$.

\section{Alternative Aufnahmewege der Medikamenteneinnahme}

Für hypothetische orale, intranasale, inhalative und subkutane Anwendungen wurde jeweils erfragt, welche Kontraindikationen für diese Applikationsformen vorlagen, ob dieser Weg jemals bei Durchbruchschmerz oder anderen medizinischen Problemen genutzt wurde, ob es Schwierigkeiten bei der Anwendung gab und ob bzw. warum Patienten diese Anwendungsform nicht in Betracht ziehen würden. Hier zeigte sich, dass $\mathrm{Pa}$ tienten bereits häufig Erfahrungen mit oralen und subkutanen Präparaten gemacht haben und diese Aufnahmewege auch für sich in Betracht ziehen, während eine tatsächliche oder hypothetische Anwendung intranasal oder inhalativ zu ap-

Tab. 1 Häufigkeiten der regelmäßigen/primären und zusätzlichen Analgesie sowie nicht-medikamentöse Maßnahmen zur Linderung des Dauer- und Durchbruchschmerzes

\begin{tabular}{|c|c|c|c|c|}
\hline $\begin{array}{l}\text { Schmerz- } \\
\text { typ }\end{array}$ & Anwendung & Analgesie & $\begin{array}{l}\text { Absolute } \\
\text { Häufigkeit } \\
(n=80)\end{array}$ & $\begin{array}{l}\text { Relative } \\
\text { Häufigkeit } \\
\text { (\%) }\end{array}$ \\
\hline \multirow{17}{*}{$\begin{array}{l}\text { Dauer- } \\
\text { schmerz }\end{array}$} & \multirow{9}{*}{$\begin{array}{l}\text { Regelmäßige } \\
\text { Analgesie }\end{array}$} & Fentanyl & 33 & 41,3 \\
\hline & & Hydromorphon & 28 & 35,0 \\
\hline & & Morphin & 12 & 15,0 \\
\hline & & Oxycodon & 2 & 2,5 \\
\hline & & Levomethadon & 1 & 1,3 \\
\hline & & Tramadol & 1 & 1,3 \\
\hline & & Fentanyl + Morphin (K) & 1 & 1,3 \\
\hline & & Hydromorphon + Tramadol (K) & 1 & 1,3 \\
\hline & & Fehlende Angaben & 1 & 1,3 \\
\hline & \multirow{8}{*}{$\begin{array}{l}\text { Zusätzliche } \\
\text { andere } \\
\text { Analgesie }\end{array}$} & NSAR & 62 & 77,5 \\
\hline & & Keine & 18 & 22,5 \\
\hline & & Antikonvulsiva & 17 & 21,3 \\
\hline & & Antidepressiva & 7 & 8,8 \\
\hline & & Neuroleptika & 2 & 2,5 \\
\hline & & Muskelrelaxanzien & 2 & 2,5 \\
\hline & & Kortikosteroide & 1 & 1,3 \\
\hline & & Benzodiazepine & 1 & 1,3 \\
\hline \multirow{29}{*}{$\begin{array}{l}\text { Durch- } \\
\text { bruch- } \\
\text { schmerz }\end{array}$} & \multirow{11}{*}{$\begin{array}{l}\text { Primäre } \\
\text { Analgesie }\end{array}$} & Morphin & 29 & 36,3 \\
\hline & & Hydromorphon & 26 & 32,5 \\
\hline & & Fentanyl (oral tm) & 10 & 12,5 \\
\hline & & Fentanyl + Morphin (K) (oral tm) & 4 & 5,0 \\
\hline & & Fehlende Angaben & 4 & 5,0 \\
\hline & & Oxycodon & 2 & 2,5 \\
\hline & & Morphin + Tramadol (K) & 1 & 1,3 \\
\hline & & Fentanyl + Hydromorphon (K) (oral tm) & 1 & 1,3 \\
\hline & & Tramadol & 1 & 1,3 \\
\hline & & Hydromorphon + Oxycodon (K) & 1 & 1,3 \\
\hline & & Levomethadon & 1 & 1,3 \\
\hline & \multirow{7}{*}{$\begin{array}{l}\text { Zusätzliche } \\
\text { Analgesie }\end{array}$} & Keine & 62 & 77,5 \\
\hline & & NSAR & 12 & 15,0 \\
\hline & & Benzodiazepine & 3 & 3,8 \\
\hline & & Paracetamol & 2 & 2,5 \\
\hline & & Spasmolytika & 1 & 1,3 \\
\hline & & Neuroleptika & 1 & 1,3 \\
\hline & & Sauerstoff & 1 & 1,3 \\
\hline & \multirow{11}{*}{$\begin{array}{l}\text { Nichtmedi- } \\
\text { kamentöse } \\
\text { Anwen- } \\
\text { dungen }\end{array}$} & Keine & 29 & 36,3 \\
\hline & & Ruhe/Schlaf & 19 & 23,8 \\
\hline & & Wärme & 13 & 16,3 \\
\hline & & Lagerung/Positionswechsel & 10 & 12,5 \\
\hline & & Bewegung & 8 & 10,0 \\
\hline & & Entspannung/Visualisierung & 7 & 8,8 \\
\hline & & Reibung/Massage & 3 & 3,8 \\
\hline & & Ablenkung & 3 & 3,8 \\
\hline & & Andere (Polsterung, Selbsthypnose, Musik) & 3 & 3,8 \\
\hline & & Kälte & 1 & 1,3 \\
\hline & & Fehlende Angaben & 1 & 1,3 \\
\hline
\end{tabular}

plizierender Medikamente eher als unangenehm empfunden und damit abgelehnt wurde (- Tab. 3).

\section{Diskussion}

Die hohe Prävalenz von Durchbruchschmerzen und die damit verbunde- nen pharmakologischen Herausforderungen aktivierten in den letzten $2 \mathrm{Jahr}$ zehnten zahlreiche Studien, die die klinischen Charakteristika des Durchbruchschmerzes genauer definieren und klare Therapieempfehlungen entwickeln sollten [13]. In dieser Arbeit wurden das Erleben von Durchbruchschmerzen aus der Sicht 
Tab. 2 Häufigkeiten der Ranglistenplätze von erwünschten Charakteristika einer Analgesie von Durchbruchschmerzen aus einer Liste von 6 vorgegebenen Merkmalen plus einer Freitextoption $(n=80)$

\begin{tabular}{|c|c|c|c|c|c|c|}
\hline & Rang 1 & Rang 2 & Rang 3 & Rang 4 & Rang 5 & Rang 6 \\
\hline Vollständige Schmerzlinderung & 48 & 16 & 8 & 4 & 1 & 0 \\
\hline Schnelle Schmerzlinderung & 22 & 43 & 9 & 3 & 0 & 0 \\
\hline Verursacht wenige Nebenwirkungen & 4 & 14 & 36 & 10 & 13 & 0 \\
\hline Praktisch/einfache Anwendung & 1 & 0 & 18 & 48 & 9 & 1 \\
\hline $\begin{array}{l}\text { Verabreichung durch Familienmit- } \\
\text { glied/Pflegepersonal möglich }\end{array}$ & 2 & 3 & 4 & 10 & 51 & 7 \\
\hline Preiswerte Therapie & 0 & 1 & 2 & 2 & 3 & 69 \\
\hline $\begin{array}{l}\text { Andere (z. B. tragbar, guter } \\
\text { Geschmack, leichter Zugang, klein) }\end{array}$ & 3 & 3 & 3 & 3 & 3 & 3 \\
\hline
\end{tabular}

der Patienten sowie erste Erfahrungen mit existierenden Therapiestrategien und Erwartungen von Patienten aus Deutschland an ein optimales, zukünftiges Schmerzmedikament untersucht.

Die hier vorgestellten Ergebnisse zu Häufigkeit, auslösenden Faktoren, Dauer und Einschränkungen durch Durchbruchschmerzen decken sich weitestgehend mit der vorliegenden Literatur [12]. Die Ergebnisse verdeutlichen den Leidensdruck der Patienten und unterstreichen die Bedeutung und Dringlichkeit der Entwicklung von weiteren adäquaten Therapiemaßnahmen zur Behandlung von Durchbruchschmerz [27].

Bei der genauen Betrachtung der Ergebnisse zu Therapien zur Dauer- und Durchbruchanalgesie fallen mehrere Besonderheiten auf. Obwohl Morphin als der Goldstandard in der Tumorschmerztherapie gewertet wird und noch vor wenigen Jahren vorrangig zum Einsatz kam [14, 20], scheint der Anteil von Patienten mit Morphin zur Daueranalgesie in dieser Erhebung eher gering zu sein zugunsten einer höheren Repräsentation von Hydromorphon und Fentanyl. Dies mag einerseits an der praktikablen Applikation als transdermales Pflaster und der daraus resultierenden Präferenz von Behandlern oder Patienten liegen, andererseits an intensiv geführtem Marketing.

Eher selten wird in der Durchbruchanalgesie Gebrauch von Fentanyl gemacht, obwohl dieses im Erhebungszeitraum als bukkale, oral transmukosal und in klinischen Studien oder als Rezepturarzneimittel auch für die intranasale Applikationsform verfügbar war. Stattdessen wurde vereinzelt sogar Gebrauch von niedrig potenten Präparaten der WHO-Stufe II (z. B. Tramadol) oder solchen mit verzögertem Wirkeintritt und langer Halbwertszeit (z. B. Oxycodon, Levomethadon) gemacht, die in der Durchbruchanalgesie nach heutigem Kenntnisstand keinen optimalen Behandlungserfolg versprechen [9].

Obwohl viele Studienteilnehmer neben ihrer medikamentösen Behandlung der Durchbruchschmerzen zusätzlich nicht-pharmakologische Maßnahmen, wie z. B. Ruhe/Schlaf, Wärme oder Lagerung/Positionswechsel zur Linderung beschrieben [22], waren nur zwei Drittel der Befragten mit der Therapie ihrer Durchbruchschmerzen tatsächlich zufrieden. Da diese Erhebung im klinischen Setting erfolgte, ist anzunehmen, dass die Therapie des Durchbruchschmerzes außerhalb spezialisierter Einrichtungen unter noch weniger optimalen Bedingungen verlaufen könnte. Auf die Frage nach den Wünschen nannten Patienten erwartungsgemäß v. a. eine vollständige und schnelle Schmerzlinderung mit wenigen Nebenwirkungen und praktikabler Eigenanwendung und legten eher wenig Wert z. B. auf die einfache Verabreichung durch andere Personen oder die Wirtschaftlichkeit der Medikamente. Diese Erwartungen wurden jedoch nicht umfassend erfüllt.

Es scheint Barrieren in der Durchbruchanalgesie zu geben, die den zufriedenstellenden Therapieerfolg für Betroffene verhindern [7]. Einerseits könnte die Wirksamkeit der Präparate durch Patienten missverstanden werden, sodass die Bedarfsmedikation bei nicht ausreichender subjektiver Schmerzlinderung nach der ersten Verabreichung nicht wiederholt wurde. Andererseits könnte schmerzbedingter Stress bei nicht ausreichender Wirkung der Durchbruchanalgesie bestehende Episoden verstärken oder gar neue Episoden auslösen, was dann als nicht zufriedenstellende Wirkung der Durchbruchanalgesie wahrgenommen wird. Letztlich können die Aussagen aus Patientensicht auch falsche Angaben enthalten, wie z. B. der Hinweis auf eine inhalative Applikation von Durchbruchanalgetika, die zum Zeitpunkt der Studiendurchführung in Deutschland nicht verfügbar waren.

Überraschend war eine wesentliche Abweichung in der deutschen Stichprobe im Vergleich zu den anderen teilnehmenden EU-Ländern bei der Beurteilung und Akzeptanz von alternativen Applikationswegen (- Tab. 3). Die deutsche Frage „Haben Sie die Verwendung solcher Präparate zur Behandlung der Schmerzverstärkungen jemals in Betracht gezogen?" wurde für die Verwendung von intranasalen und inhalativen Präparaten von fast der Hälfte der deutschen Studienteilnehmer verneint, in den anderen EU-Ländern jedoch nur von $16-26 \%$ bzw. 9-27\% der Studienteilnehmer [2, 3]. Eine ähnlich hohe Akzeptanz von oralen, intranasalen und subkutanen Applikationsformen von Durchbruchanalgetika findet sich bei bis zu 90\% aller Befragten in einer britischen Studie mit 100 Tumorpatienten [24]. Als Hauptgrund für die Ablehnung wurde von den deutschen Patienten in der vorliegenden Studie mehrheitlich angegeben, dass sie den Gedanken an solche Produkte nicht mögen, Zweifel an der Wirksamkeit dieser Produkte hegen oder Probleme mit Nase bzw. Lunge hätten, was diesem Aufnahmeweg widersprechen würde. An dieser Stelle mag spekuliert werden, dass moderne, intranasale und inhalative Applikationswege möglicherweise durch die individuelle medizinische Sozialisation von Patienten als weniger wirksame Präparate eingeschätzt werden und eher Skepsis hervorrufen. Traditionelle orale Medikationen via Tabletten oder subkutane Präparate via Injektion werden hingegen durch persönliche Erfahrungen als nachweisbar wirksam erlebt und daher eher ernst genommen.

Diese Unterschiede können jedoch am Wortlaut der Übersetzung liegen. Im eng- 
Tab. 3 Häufigkeiten von regelmäßigen Beschwerden (Mehrfachantworten möglich), Erfahrung im Gebrauch bei Durchbruchschmerz und für andere Indikationen, Schwierigkeiten bei der Anwendung, mögliche Anwendung und Ablehnungsgründe (Mehrfachantworten möglich) von oralen, intranasalen, inhalativen und subkutanen Medikamenten $(n=80)$

\begin{tabular}{|c|c|c|c|c|}
\hline & Orale Aufnahme & Intranasale Aufnahme & Inhalative Aufnahme & Subkutane Aufnahme \\
\hline Regelmäßige Beschwerden & $\begin{array}{l}\text { - Trockener Mund (51) } \\
\text { - Wunder Mund (14) } \\
\text { - Erschwertes Mundöffnen (9) } \\
\text { - Andere, z. B. Störungen des } \\
\text { Geschmacks (4) }\end{array}$ & $\begin{array}{l}\text { - Trockene Nase (3) } \\
\text { - Wunde Nase (16) } \\
\text { - Verstopfte Nase (10) } \\
\text { - Laufende Nase (8) } \\
\text { - Andere, z. B. Bluten (5) }\end{array}$ & $\begin{array}{l}\text { - Husten (19) } \\
\text { - Kurzatmigkeit (35) } \\
\text { - Keuchen (7) } \\
\text { - Andere, z. B. Schmerz } \\
\text { (1) }\end{array}$ & $\begin{array}{l}\text { - Blutergüsse (19) } \\
\text { - Blutungen (3) } \\
\text { - Empfindliche Haut (17) } \\
\text { - Andere, z. B. trockene } \\
\text { Haut (8) }\end{array}$ \\
\hline \multicolumn{5}{|c|}{ Bisheriger Gebrauch bei Durchbruchschmerzen } \\
\hline$-\mathrm{Ja}$ & $31(39 \%)$ & $1(1 \%)$ & $1(1 \%)$ & $32(40 \%)$ \\
\hline- Nein & $48(60 \%)$ & $79(99 \%)$ & $79(99 \%)$ & $48(60 \%)$ \\
\hline - Fehlende Angaben & $1(1 \%)$ & 0 & 0 & 0 \\
\hline \multicolumn{5}{|c|}{ Bisheriger Gebrauch bei anderen Problemen } \\
\hline$-\mathrm{Ja}$ & $34(43 \%)$ & $26(33 \%)$ & $22(28 \%)$ & $49(61 \%)$ \\
\hline- Nein & $46(57 \%)$ & $54(67 \%)$ & $58(72 \%)$ & $31(39 \%)$ \\
\hline \multicolumn{5}{|l|}{ Schwierigkeiten bei der Anwendung } \\
\hline$-\mathrm{Ja}$ & $3(4 \%)$ & $2(2 \%)$ & $1(1 \%)$ & $12(15 \%)$ \\
\hline- Nein & $46(58 \%)$ & $24(30 \%)$ & $21(26 \%)$ & $49(61 \%)$ \\
\hline - Nicht zutreffend & $29(35 \%)$ & $54(68 \%)$ & $58(73 \%)$ & $16(20 \%)$ \\
\hline - Fehlende Angabe & $2(3 \%)$ & 0 & 0 & $3(4 \%)$ \\
\hline \multicolumn{5}{|l|}{ Verwendung in Betracht gezogen } \\
\hline$-\mathrm{Ja}$ & $41(51 \%)$ & $7(9 \%)$ & $9(11 \%)$ & $44(55 \%)$ \\
\hline- Nein & $11(14 \%)$ & $38(48 \%)$ & $33(41 \%)$ & $17(21 \%)$ \\
\hline - Vielleicht & $28(35 \%)$ & $35(43 \%)$ & $38(48 \%)$ & $19(24 \%)$ \\
\hline \multicolumn{5}{|l|}{ Gründe, warum nicht } \\
\hline - Probleme mit Mund/Nase/Lunge/Haut & 4 & 7 & 9 & 2 \\
\hline - Ablehnung des Gedankens daran & 3 & 21 & 16 & 9 \\
\hline - Schlechte Erfahrung & 0 & 1 & 1 & 2 \\
\hline - Zweifel an Wirksamkeit & 2 & 7 & 9 & 1 \\
\hline - Zweifel über Nebenwirkungen & 2 & 5 & 5 & 2 \\
\hline - Angst vor Abhängigkeit & 1 & 4 & 2 & 1 \\
\hline - Andere, z. B. Präferenz anderer & 2 & 2 & 2 & 0 \\
\hline - Fehlende Angabe & 0 & 6 & 3 & 5 \\
\hline
\end{tabular}

lischen Original lautete die Frage „Would you consider using such a product to treat exacerbations of your pain?", was mehr als das potenzielle Abwägen dieser Behandlungsoptionen gelten kann, während die deutsche Formulierung eher auf bisher schon konkret erfolgte Nachfragen, wenn solche Applikationswege vom Behandler vorgeschlagen wurden, bezogen scheint.

Die unterschiedlichen Antworten der deutschen Teilnehmer lassen deshalb nicht eindeutig auf eine stärkere Ablehnung dieser Applikationswege in Deutschland zurückschließen.

Die von den Patienten genannten Gründe für die Abneigung gegenüber intranasalen und inhalativen Präparaten könnten auch auf ein Informationsdefizit der Patienten hinweisen, zumal die theoretischen pharmakologischen Besonderheiten der transmukosal, intrana- salen oder inhalativen Applikation den Erwartungen von Schmerzpatienten in Bezug auf vollständige und rasch einsetzende Wirkung und einfache Applikationsweise ihrer Durchbruchschmerzmedikation grundsätzlich entgegenkommen könnten.

\section{Fazit für die Praxis}

Die Ergebnisse unserer Studie bestätigen die enorme Komplexität des Phänomens „Durchbruchschmerz", bei dem nicht nur die subjektive Wahrnehmung des Symptoms selbst eine ausgeprägte Streubreite bei Tumorpatienten aufweist, sondern auch die sehr unterschiedlichen Präferenzen und Erwartungen an eine effektive Therapie nebst der Vielzahl an individuellen Gründen, die dieser entgegenstehen. Ärztliche Behandlung und Be- gleitung sollte (nicht nur in der Palliativmedizin und Tumorschmerztherapie) dieser Komplexität Rechnung tragen.

\section{Korrespondenzadresse}

\section{Dr. L. Bertram}

Klinik für Palliativmedizin, RWTH Aachen

Pauwelsstr. 30, 52074 Aachen

Ibertram@ukaachen.de

Danksagung. Die Autoren bedanken sich herzlich für die Zusammenarbeit mit der Studienkoordinatorin Ms Alison Buchanan (Royal Marsden Hospital, Großbritannien).

Interessenkonflikt. Der korrespondierende Autor weist auf folgende Beziehung hin: Diese Studie wurde durch eine Forschungsfinanzierung von Nycomed unterstützt. Für keinen der Autoren besteht ein Interessenskonflikt. 


\section{Literatur}

1. Caraceni A, Martini C, Zecca E et al (2004) Breakthrough pain characteristics and syndromes in patients with cancer pain. An international survey. Palliat Med 18(3):177-183

2. Davies A, Zeppetella G, Andersen S et al (2010) Breakthrough cancer pain: A European multi-centre study of pain characteristics and patient perception of current managment strategies. in preparation

3. Davies A, Zeppetella G, Buchanan A et al (2010) Breakthrough cancer pain: A European, multicentre study of patient perception of potential managment strategies (in Vorbereitung)

4. Davies AN, Dickman A, Reid C et al (2009) The management of cancer-related breakthrough pain: recommendations of a task group of the Science Committee of the Association for Palliative Medicine of Great Britain and Ireland. Eur J Pain 13(4):331-338

5. Fortner BV, Demarco G, Irving G et al (2003) Description and predictors of direct and indirect costs of pain reported by cancer patients. J Pain Symptom Manage 25(1):9-18

6. Gomez-Batiste X, Madrid F, Moreno F et al (2002) Breakthrough cancer pain: prevalence and characteristics in patients in Catalonia, Spain. J Pain Symptom Manage 24(1):45-52

7. Hagen NA, Biondo P, Stiles C (2008) Assessment and management of breakthrough pain in cancer patients: current approaches and emerging research. Curr Pain Headache Rep 12(4):241-248

8. Hagen NA, Fisher K, Victorino C, Farrar JT (2007) A titration strategy is needed to manage breakthrough cancer pain effectively: observations from data pooled from three clinical trials. J Palliat Med 10(1):47-55

9. Lossignol DA, Dumitrescu C (2010) Breakthrough pain: progress in management. Curr Opin Oncol 22(4):302-306

10. Mercadante S, Radbruch L, Caraceni A et al (2002) Episodic (breakthrough) pain: consensus conference of an expert working group of the European Association for Palliative Care. Cancer 94(3):832-839

11. Mercadante S, Villari P, Ferrera P, Casuccio A (2004) Optimization of opioid therapy for preventing incident pain associated with bone metastases. J Pain Symptom Manage 28(5):505-510

12. Mercadante S, Zagonel V, Breda E et al (2010) Breakthrough pain in oncology: a longitudinal study. J Pain Symptom Manage (im Druck)

13. Nauck F, Eulitz N (2007) Cancer pain management. Basic therapy and treatment of breakthrough pain. Schmerz 21(4):359-370; quiz 371-372

14. Nauck F, Ostgathe C, Klaschik E et al (2004) Drugs in palliative care: results from a representative survey in Germany. Palliat Med 18(2):100-107

15. Petzke F, Radbruch L, Zech D et al (1999) Temporal presentation of chronic cancer pain: transitory pains on admission to a multidisciplinary pain clinic. J Pain Symptom Manage 17(6):391-401

16. Portenoy RK, Bruns D, Shoemaker B, Shoemaker SA (2010) Breakthrough pain in community-dwelling patients with cancer pain and noncancer pain, part 1: prevalence and characteristics. J Opioid Manag 6(2):97-108

17. Portenoy RK, Bruns D, Shoemaker B, Shoemaker SA (2010) Breakthrough pain in community-dwelling patients with cancer pain and noncancer pain, part 2: impact on function, mood, and quality of life. J Opioid Manag 6(2):109-116
18. Portenoy RK, Hagen NA (1990) Breakthrough pain: definition, prevalence and characteristics. Pain 41(3):273-281

19. Portenoy RK, Payne D, Jacobsen P (1999) Breakthrough pain: characteristics and impact in patients with cancer pain. Pain 81(1-2):129-134

20. Radbruch $L$, Elsner $F$ (2007) Pain therapy in palliative medicine. Chir Gastroeneterol 23:51-59

21. Simmonds MA (1999) Management of breakthrough pain due to cancer. Oncology (Williston Park) 13(8):1103-1108; discussion 1110:11131114

22. Soares LG, Chan VW (2007) The rationale for a multimodal approach in the management of breakthrough cancer pain: a review. Am J Hosp Palliat Care 24(5):430-439

23. Swanwick M, Haworth M, Lennard RF (2001) The prevalence of episodic pain in cancer: a survey of hospice patients on admission. Palliat Med 15(1):9-18

24. Walker G, Wilcock A, Manderson C et al (2003) The acceptability of different routes of administration of analgesia for breakthrough pain. Palliat Med 17(2):219-221

25. World Health Organisation (WHO) (2010) WHO Definition of palliative care. http://www.who.int/cancer/palliative/definition/en/ (Zugriff: September 2010)

26. William L, Macleod R (2008) Management of breakthrough pain in patients with cancer. Drugs 68(7):913-924

27. Zeppetella G (2009) Impact and management of breakthrough pain in cancer. Curr Opin Support Palliat Care 3(1):1-6

28. Zeppetella G (2010) Breakthrough pain: In: Hanks GCN, Christakis NA, Fallon M (Hrsg) Oxford textbook of palliative medicine. University Press, Oxford. S 654-661

29. Zeppetella G, O'Doherty CA, Collins S (2000) Prevalence and characteristics of breakthrough pain in cancer patients admitted to a hospice. J Pain Symptom Manage 20(2):87-92

\section{Schmerz-Sensibilisierung nicht nur bei Fibromyalgie-Patienten}

Der bei einer Fibromyalgie (FM) auftretende chronische Schmerz wird von Müdigkeit, körperlichen Beeinträchtigungen und seelischen Qualen begleitet. FM-Patienten erleben negative Emotionen stärker als gesunde Menschen. Daher liegt die Vermutung nahe, dass Wut und Traurigkeit auch umgekehrt die Schmerzen bei FM-Patientinnen eher verstärken als bei gesunden Frauen.

Forscher der Universität Utrecht untersuchten jetzt den Effekt von negativen Emotionen auf die Schmerzwahrnehmung bei Frauen mit und ohne Fibromyalgie (FM).

In der Studie sollten sich beide Gruppen von Frauen abwechselnd eine neutrale, eine wut- und eine trauerauslösende Situation ins Gedächtnis rufen. Mit Hilfe einer Bewertungsskala, welche von „überhaupt kein Schmerz" bis hin zu "unerträglicher Schmerz" reichte, wurden die Wahrnehmungsschwelle, die Schmerzschwelle und die Schmertoleranz erfasst. Negative Gefühle erhöhten sowohl den klinischen Schmerz bei FM-Patienten, als auch den induzierten Schmerz (elektrischer Strom) bei beiden Gruppen.

In einer anderen Studie konnte ein Forscherteam zeigen, dass eine individuelle kognitive Verhaltenstherapie und Übungen zur Schmerzvermeidung oder zum Ertragen des Schmerzes relativ früh nach der Diagnose heilsame Behandlungsergebnisse für Hochrisikopatienten mit FM fördern. Es werden Verbesserungen im physischen und im psychologischen Bereich erzielt. Kurzzeitige aber auch langfristige (6 Monate) Verbesserungen stellten sich ein.

Literatur:

V Middendorp H, Lumley MA, Jacobs JWG et al (2010) The Effects of Anger and Sadness on Clinical Pain Reports and ExperimentallyInduced Pain Thresholds in Women With and Without Fibromyalgia. Arthritis Care and Res 62 (10): 1370-1376
Quelle:

Wiley-VCH, Weinheim, www.wiley.de 